TRANSACTIONS OF THE

AMERICAN MATHEMATICAL SOCIETY

Volume 364, Number 1, January 2012, Pages 551-569

S 0002-9947(2011)05494-2

Article electronically published on June 29, 2011

\title{
GENERALIZED EHRHART POLYNOMIALS
}

\author{
SHENG CHEN, NAN LI, AND STEVEN V SAM
}

\begin{abstract}
Let $P$ be a polytope with rational vertices. A classical theorem of Ehrhart states that the number of lattice points in the dilations $P(n)=n P$ is a quasi-polynomial in $n$. We generalize this theorem by allowing the vertices of $P(n)$ to be arbitrary rational functions in $n$. In this case we prove that the number of lattice points in $P(n)$ is a quasi-polynomial for $n$ sufficiently large. Our work was motivated by a conjecture of Ehrhart on the number of solutions to parametrized linear Diophantine equations whose coefficients are polynomials in $n$, and we explain how these two problems are related.
\end{abstract}

\section{INTRODUCTION}

In this article we relate two problems, one from classical number theory and one from lattice point enumeration in convex bodies. Motivated by a conjecture of Ehrhart $[\mathrm{Ehr}]$ and a result of $\mathrm{Xu}[\mathrm{Xu}$, we study linear systems of Diophantine equations with a single parameter. To be more precise, we suppose that the coefficients of our system are given by polynomial functions in a variable $n$ and also that the number of solutions $f(n)$ in nonnegative integers for any given value of $n$ is finite. We are interested in the behavior of the function $f(n)$, and in particular, we prove that $f(n)$ is eventually a quasi-polynomial, i.e., there exists some period $s$ and polynomials $f_{i}(t)$ for $i=0, \ldots, s-1$ such that for $t \gg 0$, the number of solutions for $n \equiv i(\bmod s)$ is given by $f_{i}(n)$. The other side of our problem can be stated in a similar fashion: suppose that $P(n)$ is a convex polytope whose vertices are given by rational functions in $n$. Then the number of integer points inside of $P(n)$, as a function of $n$, enjoys the same properties as that of $f$ as above. We now describe in more detail some examples and the statements of our results.

1.1. Diophantine equations. As a warmup to our result, we begin with two examples. The first is a result of Popoviciu. Let $a$ and $b$ be relatively prime positive integers. We wish to find a formula for the number of nonnegative integer solutions $(x, y)$ to the equation $a x+b y=n$. For a real number $x$, let $\lfloor x\rfloor$ denote the greatest integer less than or equal to $x$, and define $\{x\}=x-\lfloor x\rfloor$ to be the

Received by the editors May 14, 2010 and, in revised form, September 6, 2010, October 8, 2010 and October 26, 2010.

2010 Mathematics Subject Classification. Primary 11D45; Secondary 11D04, 52C07, 05A16.

Key words and phrases. Ehrhart polynomials, Diophantine equations, lattice points, polytopes, quasi-polynomials.

The first author was sponsored by Project 11001064 supported by the National Natural Science Foundation of China, and Project HITC200701 supported by the Science Research Foundation in Harbin Institute of Technology.

The third author was supported by an NSF graduate fellowship and an NDSEG fellowship.

(C)2011 American Mathematical Society

Reverts to public domain 28 years from publication 
fractional part of $x$. Then the number of such solutions is given by the formula

$$
\frac{n}{a b}-\left\{\frac{n a^{-1}}{b}\right\}-\left\{\frac{n b^{-1}}{a}\right\}+1,
$$

where $a^{-1}$ and $b^{-1}$ satisfy $a a^{-1} \equiv 1(\bmod b)$ and $b b^{-1} \equiv 1(\bmod a)$. See $\mathrm{BR}$, Chapter 1] for a proof. In particular, this function is a quasi-polynomial in $n$.

For the second example, which is a generalization of the first example, consider the number of solutions $(x, y, z) \in \mathbf{Z}_{\geq 0}^{3}$ to the matrix equation

$$
\left(\begin{array}{lll}
x_{1} & x_{2} & x_{3} \\
y_{1} & y_{2} & y_{3}
\end{array}\right)\left(\begin{array}{l}
x \\
y \\
z
\end{array}\right)=\left(\begin{array}{l}
m_{1} \\
m_{2}
\end{array}\right)
$$

where the $x_{i}$ and $y_{i}$ are fixed positive integers and $x_{i+1} y_{i}<x_{i} y_{i+1}$ for $i=1,2$. Write $Y_{i j}=x_{i} y_{j}-x_{j} y_{i}$. We assume that $\operatorname{gcd}\left(Y_{12}, Y_{13}, Y_{23}\right)=1$, so that there exist integers (not unique) $f_{i j}, g_{i j}$ such that

$$
\begin{aligned}
& \operatorname{gcd}\left(f_{12} Y_{13}+g_{12} Y_{23}, Y_{12}\right)=1, \\
& \operatorname{gcd}\left(f_{13} Y_{12}+g_{13} Y_{23}, Y_{13}\right)=1, \\
& \operatorname{gcd}\left(f_{23} Y_{13}+g_{23} Y_{12}, Y_{23}\right)=1 .
\end{aligned}
$$

Now define two regions $\Omega_{i}=\left\{(x, y) \mid \frac{y_{i}}{x_{i}}<\frac{y}{x}<\frac{y_{i+1}}{x_{i+1}}\right\}$ for $i=1,2$. Then if $m=\left(m_{1}, m_{2}\right) \in \mathbf{Z}^{2}$ is in the positive span of the columns of the matrix in (1.2), there exist Popoviciu-like formulas for the number of solutions of (1.2) which depend only on whether $m \in \Omega_{1}$ or $m \in \Omega_{2}$ and on the numbers $Y_{i j}, f_{i j}, g_{i j}, x_{i}, y_{i}$. See Section 5.1 for the precise statement.

In particular, one can replace the $x_{i}, y_{i}$, and $m_{i}$ by polynomials in $n$ in such a way that for all values of $n$, the condition $\operatorname{gcd}\left(Y_{12}, Y_{13}, Y_{23}\right)=1$ holds. For a concrete example, consider the system

$$
\left(\begin{array}{ccc}
2 n+1 & 3 n+1 & n^{2} \\
2 & 3 & n+1
\end{array}\right)\left(\begin{array}{l}
x \\
y \\
z
\end{array}\right)=\left(\begin{array}{c}
3 n^{3}+1 \\
3 n^{2}+n-1
\end{array}\right) .
$$

Then for $n \gg 0$, we have that

$$
\frac{3}{3 n+1}<\frac{3 n^{2}+n-1}{3 n^{3}+1}<\frac{n+1}{n^{2}},
$$

so that for these values of $n$, there exists a quasi-polynomial that counts the number of solutions $(x, y, z)$.

Given these examples, we are ready to state our general theorem. We denote by $\mathbf{Q P}_{\gg 0}$ the set of functions $f: \mathbf{Z} \rightarrow \mathbf{Z}$ which are eventually quasi-polynomial.

Theorem 1.1. Let $A(n)$ be an $m \times k$ matrix, and $b(n)$ be a column vector of length $m$, such that their entries are integer coefficient polynomials in $n$. If $f(n)$ denotes the number of nonnegative integer vectors $x$ satisfying $A(n) x=b(n)$ (assuming that these values are finite), then $f \in \mathbf{Q P}_{\gg 0}$.

This theorem generalizes the conjecture [Sta, Exercise 4.12]. See [Ehr, p. 139] for some verified cases of a conjectural multivariable analogue, which we state here. Let $S \subset \mathbf{Z}^{r}$ be some subset. We say that a function $f: S \rightarrow \mathbf{Z}$ is a multivariate quasi-polynomial if there exists a finite index sublattice $L \subset \mathbf{Z}^{r}$ such that $f$ is a polynomial function on each coset of $L$ intersected with $S$. 
Conjecture 1.2 (Ehrhart). Let $A\left(n_{1}, \ldots, n_{r}\right)$ be an $m \times k$ matrix and $b\left(n_{1}, \ldots, n_{r}\right)$ be a column vector of length $m$, such that all entries are linear functions in $n_{1}, \ldots, n_{r}$ with integer coefficients such that for all $n_{1}, \ldots, n_{r}$, the number of nonnegative integer solutions $x$ to $A\left(n_{1}, \ldots, n_{r}\right) x=b\left(n_{1}, \ldots, n_{r}\right)$ is finite. Then there exist finitely many polyhedral regions $R_{1}, \ldots, R_{N}$ covering $\mathbf{R}_{\geq 0}^{r}$ such that $f$ is a multivariate quasi-polynomial when restricted to each $R_{i}$.

1.2. Lattice point enumeration. We first recall a classical theorem due to Pick. Let $P \subset \mathbf{R}^{2}$ be a convex polygon with integral vertices. If $A(P), I(P)$, and $B(P)$ denote the area of $P$, the number of integer points in the interior of $P$, and the number of integer points on the boundary of $P$, respectively, then one has the equation

$$
A(P)=I(P)+\frac{1}{2} B(P)-1 .
$$

Now let us examine what happens with dilates of $P$ : define $n P=\{n x \mid x \in P\}$. Then of course $A(n P)=A(P) n^{2}$ and $B(n P)=n B(P)$ whenever $n$ is a positive integer, so we can write

$$
A(P) n^{2}=I(n P)+\frac{1}{2} B(P) n-1
$$

or equivalently,

$$
\#\left(n P \cap \mathbf{Z}^{2}\right)=I(n P)+B(n P)=A(P) n^{2}+\frac{1}{2} B(P) n+1,
$$

which is a polynomial in $n$. The following theorem of Ehrhart says that this is always the case independent of the dimension, and we can even relax the integral vertex condition to rational vertices:

Theorem 1.3 (Ehrhart). Let $P \subset \mathbf{R}^{d}$ be a polytope with rational vertices. Then the function $L_{P}(n)=\#\left(n P \cap \mathbf{Z}^{d}\right)$ is a quasi-polynomial of degree $\operatorname{dim} P$. Furthermore, if $D$ is an integer such that $D P$ has integral vertices, then $D$ is a period of $L_{P}(n)$. In particular, if $P$ has integral vertices, then $L_{P}(n)$ is a polynomial.

Proof. See [Sta, Theorem 4.6.25] or [BR, Theorem 3.23].

The function $L_{P}(t)$ is called the Ehrhart quasi-polynomial of $P$. One can see this as saying that if the vertices of $P$ are $v_{i}=\left(v_{i 1}, \ldots, v_{i d}\right)$, then the vertices of $n P$ are given by the linear functions $v_{i}(n)=\left(v_{i 1} n, \ldots, v_{i d} n\right)$. We generalize this as

Theorem 1.4. Given polynomials $v_{i j}(x), w_{i j}(x) \in \mathbf{Z}[x]$ for $0 \leq i \leq s$ and $1 \leq j \leq$ $d$, let $n$ be a positive integer such that $w_{i j}(n) \neq 0$ for all $i, j$. This is satisfied by $n$ sufficiently large, so we can define a rational polytope

$$
P(n)=\operatorname{conv}\left(p^{0}(n), p^{1}(n), \ldots, p^{s}(n)\right) \in \mathbf{R}^{d},
$$

where $p^{i}(n)=\left(\frac{v_{i 1}(n)}{w_{i 1}(n)}, \ldots, \frac{v_{i d}(n)}{w_{i d}(n)}\right)$. Then $\#\left(P(n) \cap \mathbf{Z}^{d}\right) \in \mathbf{Q P}_{\gg 0}$.

We call the function $\#\left(P(n) \cap \mathbf{Z}^{d}\right)$ a generalized Ehrhart polynomial.

In Section 2, we explain the equivalence of the two problems just mentioned and then prove our main result Theorem 1.1 in Section 3. The proof gives us an algorithm to compute these generalized Ehrhart polynomials, but it could be very complicated in practice. For computational reasons, we introduce the notions of generalized division and generalized gcd for the ring $\mathbf{Z}[x]$ in Section 4, In a sense, 
these generalize the usual notions of division and gcd for the ring of integers $\mathbf{Z}$. The methods and algorithms are quite similar, but they are more involved due to technical complications. The proofs for the correctness of the algorithms are given in this section. As an application of these tools, in Section 5 we will describe explicit computations of some special generalized Ehrhart polynomials.

\section{Equivalence of the TwO PROBlems}

As we shall see, the two problems of the Diophantine equations and lattice point enumeration are closely intertwined. In this section, we want to show that Theorem 1.1 is equivalent to Theorem 1.4. Before this, let us see the equivalence of Theorem 1.4 with the following result. For notation, if $x$ and $y$ are vectors, then $x \geq y$ if $x_{i} \geq y_{i}$ for all $i$.

Theorem 2.1. For $n \gg 0$, define a rational polytope $P(n)=\left\{x \in \mathbf{R}^{d} \mid V(n) x \geq\right.$ $c(n)\}$, where $V$ is an $r \times d$ matrix and $c$ is an $r \times 1$ column vector, both of whose entries are integer coefficient polynomials. Then $\#\left(P(n) \cap \mathbf{Z}^{d}\right) \in \mathbf{Q P}_{\gg 0}$.

Notice that the difference between Theorem 1.4 and Theorem 2.1 is that one defines a polytope by its vertices and the other by hyperplanes. So we will show their equivalence by presenting a generalized version of the algorithm connecting "vertex description" and "hyperplane description" of a polytope.

The connection is based on the fact that we can compare two rational functions $f(n)$ and $g(n)$ when $n$ is sufficiently large. For example, if $f(n)=n^{2}-4 n+1$ and $g(n)=5 n$, then $f(n)>g(n)$ for all $n>9$; we denote this by $f(n)>$ even $g(n)$ ("even" being short for "eventually"). Therefore, given a point and a hyperplane, we can test their relative position. To be precise, let $p(n)=\left(r_{1}(n), \ldots, r_{k}(n)\right)$ be a point where the $r_{i}(n)$ are rational functions and let $F(x, n)=a_{1}(n) x_{1}+a_{2}(n) x_{2}+$ $\cdots+a_{k}(n) x_{k}=0$ be a hyperplane where all the $a_{i}(n)$ are polynomials of $n$. Then exactly one of the following will be true:

$$
F(p, n)=_{\text {even }} 0, \quad F(p, n)>_{\text {even }} 0, \quad F(p, n)<_{\text {even }} 0 .
$$

Given this, we can make the following definition. We say that two points $p(n)$ and $q(n)$ lie (resp., weakly lie) on the same side of $F(p, n)$ if $F(p, n) F(q, n)>_{\text {even }} 0$ (resp., $\left.F(p, n) F(q, n) \geq_{\text {even }} 0\right)$.

2.1. Equivalence of Theorem 1.4 and Theorem 2.1, Going from the "vertex description" to the "hyperplane description":

Given all vertices of a polytope $P(n)$ whose coordinates are all rational functions of $n$, we want to get its "hyperplane description" for $n \gg 0$. Let $F(x, n)$ be a hyperplane defined by a subset of vertices. If all vertices lie weakly on one side of $F(x, n)$, we will keep it together with $\geq 0$ or $\leq 0$ or $=0$, indicating the relative position of this hyperplane and the polytope. We can get all the hyperplanes defining the polytope by this procedure.

Going from the "hyperplane description" to the "vertex description":

Let $P(n)=\left\{x \in \mathbf{R}^{d} \mid V(n) x \geq c(n)\right\}$ be a polytope, where $V$ is an $r \times d$ matrix and $c$ is an $r \times 1$ column vector, both of whose entries are integer coefficient polynomials. We want to find its vertex description. Let $f_{1}(n), \ldots, f_{r}(n)$ be the linear functionals defined by the rows of $V(n)$. So we can rewrite $P(n)$ as

$$
P(n)=\left\{x \in \mathbf{R}^{d} \mid\left\langle f_{i}(n), x\right\rangle \geq c_{i}(n) \text { for all } i\right\} .
$$


The vertices of $P(n)$ can be obtained as follows. For every $d$-subset $I \subseteq\{1, \ldots, r\}$, if the equations $\left\{\left\langle f_{i}(n), x\right\rangle=c_{i}(n) \mid i \in I\right\}$ are linearly independent for $n \gg 0$ and their intersection is nonempty, then it consists of a single point, which we denote by $v_{I}(n)$. If $\left\langle f_{j}(n), v_{I}(n)\right\rangle \geq c_{j}(n)$ for all $j$, then $v_{I}(n) \in \mathbf{Q}(n)^{d}$ is a vertex of $P(n)$, and all vertices are obtained in this way. We claim that the subsets $I$ for which $v_{I}(n)$ is a vertex remains constant if we take $n$ sufficiently large. First, the notion of being linearly independent equations can be tested by showing that at least one of the $d \times d$ minors of the rows of $V(n)$ indexed by $I$ does not vanish. Since these minors are all polynomial functions, they can only have finitely many roots unless they are identically zero. Hence taking $n \gg 0$, we can assume that $\left\{f_{i}(n) \mid i \in I\right\}$ is either always linearly dependent or always linearly independent. Similarly, the sign of $\left\langle f_{j}(n), v_{I}(n)\right\rangle$ is determined by the sign of a polynomial and hence is constant for $n \gg 0$.

2.2. Equivalence of Theorem 1.1 and Theorem 2.1. We can easily transform an inequality to an equality by introducing some slack variables, and we can also represent an equality $f(n, x)=0$ by two inequalities $f(n, x) \geq 0$ and $-f(n, x) \geq 0$. So the main difference between the two theorems is that Theorem 1.1 is counting nonnegative solutions while Theorem 2.1 is counting all integral solutions. But we can deal with this by adding constraints on each variable.

A more interesting connection between Theorem 1.1 and Theorem 2.1 is worth mentioning here. First consider any fixed integer $n$. Then the entries of $A(n)$ and $b(n)$ in the linear Diophantine equations $A(n) x=b(n)$ of Theorem 1.1 all become integers. For an integer matrix, we can calculate its Smith normal form. Similarly, we can use a generalized Smith normal form for matrices over $\mathbf{Q P} \mathbf{P}_{\gg 0}$ to get a transformation from Theorem 1.1 to Theorem 2.1

Then given $A(n)$ and $b(n)$, by Theorem 4.6, we can put $A(n)$ into generalized Smith normal form: $D(n)=U(n) A(n) V(n)$ for some matrix

$$
D(n)=\left(\operatorname{diag}\left(d_{1}(n), \ldots, d_{r}(n), 0, \ldots, 0\right) \mid \mathbf{0}\right)
$$

with nonzero entries only on its main diagonal and unimodular matrices $U(n)$ and $V(n)$. Then the equation $A(n) x=b(n)$ can be rewritten as $D(n) V(n)^{-1} x=$ $U(n) b(n)$. Set $y=V(n)^{-1} x$ and $b^{\prime}(n)=U(n) b(n)$. By the form of $D(n)$, we have a solution $y$ if and only if $d_{i}(n)$ divides $b_{i}^{\prime}(n)$ for $i=1, \ldots, r$, and for any given solution, the values $y_{r+1}, \ldots, y_{k}$ can be arbitrary. However, since $V(n) y=x$, we need to require that $V(n) y \geq 0$, and any such $y$ gives a nonnegative solution $x$ to the original problem. Simplifying $V(n) y \geq 0$, where $V(n)=\left(v_{1}(n), \ldots, v_{k}(n)\right)$, we get $V^{\prime}(n) X \geq c(n)$, where $V^{\prime}(n)=\left(v_{r+1}(n), \ldots, v_{k}(n)\right), X=\left(y_{r+1}, \ldots, y_{k}\right)$ and $c(n)=-\left(v_{1}(n) y_{1}+\cdots+v_{r}(n) y_{r}\right)$. Although $V^{\prime}(n)$ and $c(n)$ have entries in $\mathbf{Q} \mathbf{P}_{\gg 0}$, we can assume that they are polynomials by dealing with each constituent of the quasi-polynomials separately. So we reduce Theorem 1.1 to Theorem 2.1.

Example 2.2. Consider the nonnegative integer solutions to

$$
\left(\begin{array}{ll}
n^{2}+2 n & 2 n+2
\end{array}\right)\left(\begin{array}{l}
x_{1} \\
x_{2}
\end{array}\right)=(2 n+4)\left(n^{2}+2 n\right) .
$$

Write $A(n)=\left(\begin{array}{ll}n^{2}+2 n & 2 n+2\end{array}\right)$. When $n=2 m$, the Smith normal form of $A(2 m)$ is

$$
\left(\begin{array}{ll}
4 m^{2}+4 m & 4 m+4
\end{array}\right)\left(\begin{array}{cc}
1 & -1 \\
-(m-1) & m
\end{array}\right)=\left(\begin{array}{ll}
4 m+4 & 0
\end{array}\right) .
$$


Therefore, equation (2.1) becomes

$$
\left(\begin{array}{ll}
4 m+4 & 0
\end{array}\right)\left(\begin{array}{cc}
1 & -1 \\
-(m-1) & m
\end{array}\right)^{-1}\left(\begin{array}{l}
x_{1} \\
x_{2}
\end{array}\right)=(2 n+4)\left(n^{2}+2 n\right) .
$$

Set $\left(\begin{array}{cc}1 & -1 \\ -(m-1) & m\end{array}\right)^{-1}\left(\begin{array}{l}x_{1} \\ x_{2}\end{array}\right)=\left(\begin{array}{l}y_{1} \\ y_{2}\end{array}\right)$ so that we have

$$
\left(\begin{array}{ll}
4 m+4 & 0
\end{array}\right)\left(\begin{array}{l}
y_{1} \\
y_{2}
\end{array}\right)=(2 n+4)\left(n^{2}+2 n\right),
$$

and thus $y_{1}=n^{2}+2 n$. By the condition $\left(\begin{array}{l}x_{1} \\ x_{2}\end{array}\right) \geq 0$, we require

$$
\left(\begin{array}{cc}
1 & -1 \\
-(m-1) & m
\end{array}\right)\left(\begin{array}{c}
n^{2}+2 n \\
y_{2}
\end{array}\right) \geq 0
$$

which gives us

$$
\frac{(m-1)\left(n^{2}+2 n\right)}{m} \leq y_{2} \leq n^{2}+2 n,
$$

a one dimensional polytope. So the number of solutions for $n=2 m$ is $f(n)=2 n+5$.

We can consider the case $n=2 m+1$ similarly. The Smith normal form of $A(2 m+1)$ is

$$
\left(\begin{array}{ll}
4 m^{2}+8 m+2 & 4 m+6
\end{array}\right)\left(\begin{array}{cc}
1 & -2 \\
-m & 2 m+1
\end{array}\right)=\left(\begin{array}{l}
2 m+3 \\
-m
\end{array}\right)
$$

and $\frac{2 m\left(n^{2}+2 n\right)}{2 m+1} \leq y_{2} \leq n^{2}+2 n$, thus $f(n)=n+3$.

The proof of Theorem 4.6 is based on a theory of generalized division and GCD over the ring $\mathbf{Z}[x]$, which mainly says that for $f(x), g(x) \in \mathbf{Z}[x]$, the functions $\left\lfloor\frac{f(n)}{g(n)}\right\rfloor,\left\{\frac{f(n)}{g(n)}\right\}$, and $\operatorname{gcd}(f(n), g(n))$ lie in the ring $\mathbf{Q} \mathbf{P}_{\gg 0}$. One interesting consequence of these results is that every finitely generated ideal in $\mathbf{Q P} \mathbf{P}_{\gg 0}$ is principal, despite the fact that $\mathbf{Q P} \mathbf{P}_{\gg 0}$ is not Noetherian. We developed this theory in order to appoach Theorem 1.1 at first but subsequently have found a proof that circumvents its use. Further details can be found in Section 4 .

\section{Proof of Theorem 1.1}

To prove Theorem 1.1 we will use a "writing in base $n$ " trick to reduce equations with polynomial coefficients to linear functions. Briefly, the idea of the following "writing in base $n$ " trick is as follows: given a linear Diophantine equation

$$
a_{1}(n) x_{1}+a_{2}(n) x_{2}+\cdots+a_{k}(n) x_{k}=m(n)
$$

with polynomial coefficients $a_{i}(n)$ and $m(n)$, fix an integer $n$; then the coefficients all become integers. Now consider a solution $\left(x_{1}, x_{2}, \ldots, x_{k}\right)$ with $x_{i} \in \mathbf{Z}_{\geq 0}$. Put the values of $\left(x_{1}, x_{2}, \ldots, x_{k}\right)$ into the equation; then both sides become an integer. Then we use the fact that any integer has a unique representation in base $n$ ( $n$ is a fixed number), and we compare the coefficient of each power of $n$ on both sides of the equation.

Finally, letting $n$ change, we happen to have a uniform expression for both sides in base $n$ when $n$ is sufficiently large. Moreover, the coefficient of each power of $n$ on both sides of the equation are all linear functions of $n$ (Lemma 3.1 and Lemma 3.2). Then by Lemma 3.3, we can reduce these equations with linear function 
coefficients to the case when we can apply Ehrhart's theorem (Theorem [1.3) to show that the number of solutions are quasi-polynomials of $n$. This completes the proof of Theorem 1.1.

Lemma 3.1. Given $p(x) \in \mathbf{Z}[x]$ with $p(n)>0$ for $n \gg 0$ (i.e., $p(x)$ has positive leading coefficient), there is a unique representation of $p(n)$ in base $n$ :

$$
p(n)=c_{d}(n) n^{d}+\cdots+c_{1}(n) n+c_{0}(n)
$$

where $c_{i}(n)$ is a linear function of $n$ such that for $n \gg 0,0 \leq c_{i}(n) \leq n-1$ for $i=0,1, \ldots, d$ and $0<c_{d}(n) \leq n-1$. We denote $d=\operatorname{deg}_{n}(p(n))$.

Note that $\operatorname{deg}_{n}(p(n))$ may not be equal to $\operatorname{deg}(p(n))$. For example, $n^{2}-n+3$ is represented as $c_{1}(n) n+c_{0}(n)$ with $d=1, c_{1}(n)=n-1$, and $c_{0}(n)=3$.

Proof. Let $p(x)=a_{\ell} x^{\ell}+\cdots+a_{1} x+a_{0}$ with integral coefficients and $a_{\ell}>0$. If the $a_{i}$ are all nonnegative, the proposition holds for $n>\max _{0 \leq i \leq \ell}\left\{a_{i}\right\}$. Otherwise we can prove it by induction on $\ell-i$, where $i$ is the smallest index such that $a_{i}<0$. Suppose $i_{0} \in\{0, \ldots, \ell-1\}$ is the minimal number such that $a_{i_{0}}<0$. Then in the representation of $p(n)$, put $c_{j}=a_{j}$ for $j<i_{0}, c_{i_{0}}=n-a_{i_{0}}$. By induction, we have a representation for

$$
p(n)-\left(n+a_{i_{0}}\right) n^{i_{0}}-a_{i_{0}-1} n^{i_{0}-1}-\cdots-a_{0},
$$

so we can add it to $\left(n+a_{i_{0}}\right) n^{i_{0}}+\cdots+a_{0}$ to get the desired representation for $p(n)$. Since for $n \gg 0$ we have $c_{i_{0}}=n+a_{i_{0}}>0$ by induction, we can make sure $0 \leq c_{i} \leq n-1$ for $n \gg 0$. For a lower bound, $C=\max _{0 \leq i \leq \ell}\left\{\left|a_{i}\right|\right\}+1$ is sufficient, i.e., for all $n \geq C$, the desired unique representation is guaranteed to exist. Note that $i_{0} \neq \ell$, since $a_{\ell}>0$. So this process will stop in finitely many steps. Uniqueness of this representation is clear.

Lemma 3.2. Fix an integer $N$ and $n \gg 0$. Consider the set

$$
\begin{aligned}
S_{1}(n)=\left\{\left(x_{1}, \ldots, x_{k}\right) \in \mathbf{Z}^{k} \mid\right. & 0 \leq x_{i}<n^{N+1}, \\
& \left.a_{1}(n) x_{1}+a_{2}(n) x_{2}+\cdots+a_{k}(n) x_{k}=m(n)\right\},
\end{aligned}
$$

where $a_{i}(n)=\sum_{\ell=0}^{d_{i}} a_{i \ell} n^{\ell}$ (as a usual polynomial) and $m(n)=\sum_{\ell=0}^{d} b_{\ell} n^{\ell}$ (represented in base $n$ as in Lemma [3.1), with $b_{d} \neq 0$ and $d \geq \max _{1 \leq i \leq k}\left\{d_{i}\right\}$. Then $S_{1}(n)$ is in bijection with a finite union of sets of the form

$$
\begin{array}{r}
S_{2}(n)=\left\{0 \leq\left(x_{i j}\right)_{\substack{1 \leq i \leq k \\
0 \leq j \leq N}}<n, x_{i j} \in \mathbf{Z} \mid \text { all constraints on } x=\left(x_{i j}\right)\right. \\
\text { are of the form } A n+B=f(x)\},
\end{array}
$$

where $A, B \in \mathbf{Z}$ and $f(x)$ is a linear form of $x$ with constant coefficients.

Proof. Fix a sufficiently large positive integer $n$. By our assumptions, for any point $\left(x_{1}, \ldots, x_{k}\right) \in S_{1}(n)$ we can write $x_{i}=x_{i, N} n^{N}+x_{i, N-1} n^{N-1}+\cdots+x_{i, 0}$ with $0 \leq x_{i, j}<n$. The rest of the lemma is a direct "base $n$ " comparison starting from the lowest power to the highest power in the equation $\sum a_{i}(n) x_{i}=m(n)$. To get a feel for the proof, we recommend that the reader look at Example 3.5 first. We will use this as a running example to explain the steps of the proof.

First write $m(n)=m_{N}(n) n^{N}+m_{N-1}(n) n^{N-1}+\cdots+m_{0}(n)$ in base $n$ so that each $m_{i}(n)=m_{i}^{\prime \prime} n+m_{i}^{\prime}$ is a linear function in $n$. Then we know that $0 \leq m_{i}(n)<$ $n$ since we have fixed $n$ sufficiently large. Going back to Example 3.5, we have $m(n)=4 n^{2}+3 n-5$ so that $m_{2}(n)=4, m_{1}(n)=2$ and $m_{0}(n)=n-5$, so that 
here "sufficiently large" means $n \geq 5$. Now expand the equation $\sum a_{i}(n) x_{i}=m(n)$ to get

$$
\sum_{i=1}^{k}\left(\left(\sum_{\ell=0}^{d_{i}} a_{i \ell} n^{\ell}\right)\left(x_{i, N} n^{N}+\cdots+x_{i, 0}\right)\right)=m_{N}(n) n^{N}+\cdots+m_{0}(n) .
$$

The constant term in base $n$ of (3.1) gives us the equation

$$
a_{10} x_{1,0}+\cdots+a_{k 0} x_{k, 0}=m_{0}(n) \quad(\bmod n) .
$$

Since we have the bound $0 \leq x_{i, 0}<n$ for all $i$, we can in fact say that the LHS is equal to $m_{0}(n)+C_{0} n$, where $C_{0}$ is an integer such that $C_{0}$ is strictly greater than the sum of the negative $a_{i 0}$ and strictly less than the sum of the positive $a_{i 0}$ (and $C_{0}$ can also be 0 ). But note that $C_{0}$ only depends on the $a_{i 0}$, so if our $n$ is sufficiently large, we may assume that $\left|C_{0}\right|<n$. Going back to Example 3.5, we have the equation

$$
2 x_{10}+x_{20}=n-5 \quad(\bmod n)
$$

so that $0 \leq C_{0} \leq 2$.

Now we have to deal with finitely many cases for the value of $C_{0}$. Fix one. Going back to equation (3.1), we can substitute our value of $C_{0}$ and compare linear coefficients to get

$$
\sum_{i=1}^{k}\left(a_{i 1} x_{i, 0}+a_{i 0} x_{i, 1}\right)+C_{0}=m_{1}(n) \quad(\bmod n) .
$$

Again, since we know that $0 \leq x_{i, 0}<n$ and $0 \leq x_{i, 1}<n$ for all $i$, we can say that the LHS is equal to $m_{1}(n)+C_{1} n$, where $C_{1}$ is an integer such that $C_{1}$ is greater than the sum of the negative $a_{i 0}$ and $a_{i 1}$ and is less than the sum of the positive $a_{i 0}$ and $a_{i 1}$ (this is independent of $C_{0}$ because $\left|C_{0}\right|<n$ ). Going back to Example 3.5. we get the equation

$$
2 x_{11}+x_{21}+x_{20}+C_{0}=2(\bmod n),
$$

so $0 \leq C_{1} \leq 4$

Now we have finitely many cases of $C_{1}$, and again we fix one. We continue in this manner to get $C_{2}, C_{3}, \ldots, C_{N-1}$. At each point, we had only finitely many choices for the next $C_{i}$ so that at the end we only have finitely many sequences $C_{\bullet}=\left(C_{0}, \ldots, C_{N-1}\right)$. For a given $C_{\bullet}$, we see that the $x_{i j}$ must satisfy finitely many equalities of the form $f(x)=A n+B$, where $f$ is a linear form in $x$ with constant coefficients and $A, B \in \mathbf{Z}$. In Example 3.5, for $C_{\bullet}=(0,2)$, we would have the equations

$$
\begin{aligned}
2 x_{10}+x_{20} & =n-5, \\
2 x_{11}+x_{21}+x_{20} & =2+\underline{2} n, \\
2 x_{12}+x_{21}+x_{30}+\underline{2} & =4
\end{aligned}
$$

along with the inequalities $0 \leq x_{i j}<n$, and we have underlined the places where $C_{1}$ appears.

Each such $C_{\bullet}$ gives us a set of the form $S_{2}(n)$, and to complete the proof we take the union of these sets over all sequences $C_{\bullet}$.

We will need one more prepatory lemma before proceeding to the proof of Theorem 1.1 . 
Lemma 3.3. If $P(n)$ is a polytope defined by inequalities of the form $A n+B \leq$ $f(x)$, where $A, B \in \mathbf{Z}$ and $f(x)$ is a linear form of $x$ with constant coefficients, then $L_{P} \in \mathbf{Q P}_{\gg 0}$.

Proof. We first use the fact that the combinatorics of $P(n)$ stabilizes for sufficiently large $n$ (see Section 2); say that the polytope has $s$ vertices $v_{1}(n), \ldots, v_{s}(n)$. Also, note that the coefficients of the vertices of the polytope are linear functions in $n$ with rational coefficients. Let $D$ be the least common multiple of the denominators that appear in all of the coordinates. Then by Bar, Theorem 18.4], the function sending $\left(v_{1}(n), \ldots, v_{s}(n)\right)$ to the number of integer points in $P(n)$ is a polynomial if we restrict our attention to a specific congruence class of $n$ modulo $D$. We can compose each of these functions with the polynomial $n \mapsto\left(v_{1}(n), \ldots, v_{s}(n)\right)$ to conclude that $L_{P} \in \mathbf{Q P}_{\gg 0}$.

We can also give a self-contained proof of Lemma 3.3 that only uses Ehrhart's theorem (Theorem 1.3).

Alternate proof of Lemma 3.3. We will perform induction on dimension first, the case of dimension 0 being trivial. An inequality of the form $A n+B \leq f(x)$ with $B \neq 0$ will be called an inequality with constant term. Second, we will perform induction on the number of inequalities with constant term. Theorem 1.3 gives the desired result when there are no inequalities with constant term. In general, let $P(n)$ be a polytope defined by $A n+B \leq f(x)$ plus other relations of the same form, which we will call $R(n)$. Let $P^{\prime}(n)$ be the polytope with relations $A n \leq f(x)$ and $R(n)$. If $B>0$, then for $i=0, \ldots, B-1$, let $P_{i}(n)$ be the polytope with relations $A n+i=f(x)$ and $R(n)$. Then

$$
L_{P}(n)=L_{P^{\prime}}(n)-\sum_{i=0}^{B-1} L_{P_{i}}(n),
$$

so to show $L_{P} \in \mathbf{Q P}_{\gg 0}$ it suffices to show that $L_{P^{\prime}} \in \mathbf{Q P}_{\gg 0}$ and $L_{P_{i}} \in \mathbf{Q} \mathbf{P}_{\gg 0}$ for $i=0, \ldots, B-1$. Since $P^{\prime}(n)$ has one less inequality with constant term, we know that $L_{P^{\prime}} \in \mathbf{Q P} \mathbf{P}_{\gg 0}$ by induction. As for $P_{i}(n)$, write $f(x)=c_{1} x_{1}+\cdots+c_{k} x_{k}$ and choose $j$ such that $c_{j} \neq 0$. Then the equation $A n+i=f(x)$ can be rewritten as

$$
x_{j}=c_{j}^{-1}\left(A n+i-\sum_{\ell \neq j} c_{\ell} x_{\ell}\right) .
$$

Making substitutions into the relations $R$, each relation still has the form $A^{\prime} n+$ $B^{\prime} \leq f^{\prime}(x)$ (clear denominators if necessary to make sure all of the coefficients are integral), and we have eliminated the variable $x_{j}$.

If $B<0$, then for $i=1, \ldots,-B$, let $P_{i}(n)$ be the polytope with relations $A n+i=f(x)$ and $R(n)$. Then

$$
L_{P}(n)=L_{P^{\prime}}(n)+\sum_{i=1}^{-B} L_{P_{i}}(n)
$$

and we proceed as before. Hence $L_{P_{i}} \in \mathbf{Q P}_{\gg 0}$ by induction on dimension.

Proof of Theorem 1.1. Consider a system of linear Diophantine equations $A(n) x=$ $b(n)$ with finitely many nonnegative integer solutions for each $n \gg 0$, as in Theorem 1.1. Let

$$
a_{1}(n) x_{1}+a_{2}(n) x_{2}+\cdots+a_{k}(n) x_{k}=m(n)
$$


be any equation from the system. The equations $A(n) x=b(n)$ define a bounded polytope whose vertices are given by rational functions of $n$ as discussed in Section2, where the degrees of the rational functions (the difference between the degree of the numerator and the denominator) are independent of $n$. We can express each point in this polytope as a convex combination of the vertices. Therefore, writing each variable $x_{1}, \ldots, x_{k}$ in base $n$, we can find some positive integer $N$, which does not depend on $n$, such that the coordinates of each point in the polytope are less than $n^{N+1}$. Using Lemma 3.2 we can reduce each equation from $A(n) x=b(n)$ into a new system such as $S_{2}$, with more variables than $S_{1}$ but where all restrictions are of the form $A n+b \leq f(x)$. Applying Lemma 3.3 completes the proof.

\subsection{Examples.}

Example 3.4. We give an example for Lemma 3.3. For $n$ a positive integer, let $P(n)$ be the polygon defined by the inequalities $x \geq 0, y \geq 0$ and $-2 x-y \geq-n-1$. Then $P^{\prime}(n)$ is defined by the inequalities $x \geq 0, y \geq 0$, and $2 x+y \leq n$, and $P_{1}(n)$ is defined by the inequalities $x \geq 0, y \geq 0$, and $n+1=2 x+y$. We can rewrite the equality as $y=n+1-2 x$, and then the other inequalities become $x \geq 0$ and $n+1 \geq 2 x$.

We see that $P^{\prime}(n)$ is the convex hull of the points $\{(0,0),(0, n),(n / 2,0)\}$, while $P_{1}(n)$ is the interval $[0,(n+1) / 2]$. The total number of integer points in $P^{\prime}(n)$ and $P_{1}(n)$ is given by the quasipolynomial

$$
\#\left(P(n) \cap \mathbf{Z}^{2}\right)= \begin{cases}k^{2}+3 k+2 & \text { if } n=2 k, \\ k^{2}+4 k+4 & \text { if } n=2 k+1 .\end{cases}
$$

Its rational generating function is

$$
\sum_{n \geq 0} \#\left(P(n) \cap \mathbf{Z}^{2}\right) t^{n}=\frac{t^{5}-3 t^{3}+4 t+2}{\left(1-t^{2}\right)^{3}}=\frac{t^{3}-2 t^{2}+2}{(1-t)^{3}(1+t)} .
$$

Example 3.5. We give an example of Lemma 3.2. Consider nonnegative integer solutions for

$$
2 x_{1}+(n+1) x_{2}+n^{2} x_{3}=4 n^{2}+3 n-5 .
$$

For any $n>5$, RHS $=4 n^{2}+2 n+(n-5)$ is the expression in base $n$. Now consider the left hand side. Writing $x_{1}, x_{2}, x_{3}$ in base $n$, let $x_{1}=x_{12} n^{2}+x_{11} n+x_{10}$, $x_{2}=x_{21} n+x_{20}$ and $x_{3}=x_{30}$ with $0 \leq x_{i j}<n$. In this case, $N=2$, but we have further restrictions on the degrees of $x_{2}$ and $x_{3}$ in base $n$ coming from the coefficients $a(n)$. Then we have

$$
\text { LHS }=\left(2 x_{12}+x_{21}+x_{30}\right) n^{2}+\left(2 x_{11}+x_{21}+x_{20}\right) n+\left(2 x_{10}+x_{20}\right) .
$$

Now we can write the left hand side in base $n$ with extra constraints on the $\left(x_{i j}\right)$ 's.

We start by comparing the coefficient of $n^{0}$ on both sides. We have the following three cases:

$$
\begin{gathered}
A_{0}^{0}=\left\{2 x_{10}+x_{20}=n-5\right\}, \\
A_{1}^{0}=\left\{2 x_{10}+x_{20}=(n-5)+n\right\}
\end{gathered}
$$

and

$$
A_{2}^{0}=\left\{2 x_{10}+x_{20}=(n-5)+2 n\right\} .
$$


Therefore, in the language of the proof of Lemma 3.2 we have $0 \leq C_{0} \leq 2$. We next consider the $n^{1}$ term. If $x$ satisfies $A_{C_{0}}^{0}$ for $n^{0}, C_{0} \in\{0,1,2\}$, then the equation is reduced to

$$
\left(2 x_{12}+x_{21}+x_{30}\right) n^{2}+\left(2 x_{11}+x_{21}+x_{20}+C_{0}\right) n=4 n^{2}+2 n .
$$

Now compare the $n^{1}$ terms. We have five cases for each $C_{0} \in\{0,1,2\}$ :

$$
A_{C_{0}, C_{1}}^{1}=\left\{2 x_{11}+x_{21}+x_{20}+C_{0}=C_{1} n+2\right\},
$$

where $C_{1} \in\{0,1,2,3,4\}$.

Last, we compare the $n^{2}$ terms. Note that since we assume $n \gg 0$, the $n^{0}$ term won't carry over to the $n^{2}$ term, so the computation of the $n^{2}$ term only depends on the term $n^{1}$. If $x$ satisfies the $C_{1}$ th condition for $n^{1}$, the equation then becomes

$$
\left(2 x_{12}+x_{21}+x_{30}+C_{1}\right) n^{2}=4 n^{2} .
$$

So for each $0 \leq C_{1} \leq 4$, we have

$$
A_{C_{1}}^{2}=\left\{2 x_{12}+x_{21}+x_{30}+C_{1}=4\right\} .
$$

Overall, we have that the set

$$
\left\{\left(x_{1}, x_{2}, x_{3}\right) \in \mathbf{Z}_{\geq 0}^{3} \mid 2 x_{1}+(n+1) x_{2}+n^{2} x_{3}=4 n^{2}+3 n-5\right\}
$$

is in bijection with the set

$$
\left\{x=\left(x_{12}, x_{11}, x_{10}, x_{21}, x_{20}, x_{30}\right) \in \mathbf{Z}_{\geq 0}^{6}, 0 \leq x_{i j}<n\right\},
$$

such that $x$ satisfies the equations in the sets

$$
\left(\begin{array}{lll}
A_{0}^{0} & A_{1}^{0} & A_{2}^{0}
\end{array}\right)\left(\begin{array}{cccc}
A_{00}^{1} & A_{01}^{1} & \cdots & A_{04}^{1} \\
A_{10}^{1} & A_{11}^{1} & \cdots & A_{14}^{1} \\
A_{20}^{1} & A_{21}^{1} & \cdots & A_{24}^{1}
\end{array}\right)\left(\begin{array}{c}
A_{0}^{2} \\
A_{1}^{2} \\
\vdots \\
A_{4}^{2}
\end{array}\right) .
$$

Here we borrow the notation of matrix multiplication $A B$ to represent the intersection of sets $A \cap B$ and matrix summation $A+B$ to represent the set union $A \cup B$. Note that here all constrains $A_{i}^{j}$ on $x=\left(x_{12}, x_{11}, x_{10}, x_{21}, x_{20}, x_{30}\right)$ are in the form of $A n+B \leq f(x)$, where $A, B \in \mathbf{Z}$ and $f(x)$ is a linear form of $x$ with constant coefficients.

\section{Generalized Division}

An integer-valued polynomial, or a numerical polynomial, is a function $f: \mathbf{Z} \rightarrow \mathbf{Z}$ with a polynomial $g(x) \in \mathbf{Q}[x]$ satisfying $f(n)=g(n)$ for all $n \in \mathbf{Z}$. For any numerical polynomial $f$ and a constant $c \in \mathbf{Z}$, it is not hard to see that $\left\lfloor\frac{f(n)}{c}\right\rfloor \in$ $\mathbf{Q P}_{\gg 0}$. We want to generalize this to $\left\lfloor\frac{f(n)}{g(n)}\right\rfloor$ for two numerical polynomials $f$ and g. Based on classical division, we have for all $n \in \mathbf{Z}$ that $f(n)=\left\lfloor\frac{f(n)}{g(n)}\right\rfloor g(n)+$ $\left\{\frac{f(n)}{g(n)}\right\} g(n)$, with $0 \leq\left\{\frac{f(n)}{g(n)}\right\} g(n)$, and we define two integer-valued functions $p(n)=\left\lfloor\frac{f(n)}{g(n)}\right\rfloor$, called the quotient of $f(n)$ and $g(n)$, and $r(n)=\left\{\frac{f(n)}{g(n)}\right\} g(n)$, called the remainder of $f(n)$ and $g(n)$. We will show that $p, r \in \mathbf{Q P}_{\gg 0}$ by the generalized division algorithm.

This generalized division algorithm is based on the division for $f, g \in \mathbf{Q}[x]$, except for two modifications. First, in order to have $0 \leq r(n)<g(n)$ for $n \gg 0$, 
we need to change the remainder condition of division in $\mathbf{Q}[x]$, as in Algorithm 1 . lines 28 33. For example, for $f(x)=x-3$ and $g(x)=x$ we should have $p(x)=0$, $r(x)=x-3$, but these do not satisfy $\operatorname{deg}(r)<\operatorname{deg}(g)$ or $r=0$ for division in $\mathbf{Q}[x]$. Second, in order to keep $p(x), r(x) \in \mathbf{Z}[x]$, we break $x$ into finitely many "branches" whenever we have noninteger coefficients, as in Algorithm 1, lines 1120. For example, take $f(x)=x^{2}+3 x$ and $g(x)=2 x+1$. In trying to do division in $\mathbf{Q}[x]$, leading coefficient of $p$ will not be an integer, so we break $x$ into 2 "branches", since 2 is the leading coefficient of $g(x)$. Now we do division to $f(2 t)=4 t^{2}+6 t$ and $g(2 t)=4 t+1$ when $x=2 t$ and $f(2 t+1)=4 t^{2}+10 t+4$ and $g(2 t+1)=4 t+3$ when $x=2 t+1$. For $x=2 t$, we have $p_{0}(t)=t+1$ and $r_{0}(t)=t-1$, and for $x=2 t+1$ we have $p_{1}(t)=t+1$ and $r_{1}(t)=3 t+1$. We will show (see Theorem 4.1) that breaking $x$ into $T=b_{\ell}^{k-\ell}$ (using notation in Algorithm 1) "branches" is sufficient to conclude that $p_{i}(t), r_{i}(t) \in \mathbf{Z}[t]$, for $i=0, \ldots, T-1$.

Note that in Algorithm 1] we define lc $(r)$ to be the leading coefficient of $r$.

Theorem 4.1. Algorithm 1 is correct.

Proof. The algorithm will stop after finitely many steps $(\operatorname{deg}(r)$ is reduced by 1 in each step in line 16$)$. For $i=0, \ldots, T-1$ and for $n \equiv i(\bmod T)$, we claim the following two properties:

1. $r_{i}(x), p_{i}(x) \in \mathbf{Z}[x]$.

2. $f(T x+i)=p_{i}(x) g(T x+i)+r_{i}(x)$ and $0 \leq r_{i}(m)<g(n)$, for $n \gg 0$ and $n=T m+i$.

These two properties imply the correctness of the algorithm. By the algorithm, property 2 is obvious, so we only need to show property 1 . Note that in each step we add to $p_{i}(x)$ some $h(x)=\frac{a}{b_{\ell} T^{\ell}} x^{K-\ell}$ or an integer $c$ (see lines 17 and 23), and we subtract $h(x) g(T x+i)$ or $c g(T x+i)$ from $r_{i}(x)$ (see lines [16] and 24), so it suffices to show $h(x) \in \mathbf{Z}[x]$. For every $r_{i}(x)$ with degree $K$ in lines [1]-20, denote it by $r_{i}^{K}(x)$ and its leading coefficient by $a_{K}$. So we need to prove that $b_{\ell} T^{\ell} \mid a_{K}$ for all $K>\ell$. To show this, we use induction on $s=k-K$ to prove a stronger statement that $T^{d} \mid$ coeff $\left(x^{d}\right)$ for every monomial $x^{d}$ in $r_{i}^{K}(x)$. If $s=0$, as in line 13. $r_{i}^{K}(x)=f(T x+i)$ and the claim holds by binomial expansion. Assume it still holds for some $s$ such that $K=k-s>\ell+1$. Then for $s+1$, we have $K=k-s-1$. In line 16

$$
r_{i}^{K}(x)=r_{i}^{K+1}(x)-h(x) g(T x+i),
$$

so we only need to show that

$$
h(x) g(T x+i)=\frac{a_{K+1}}{b_{\ell} T^{\ell}} x^{k-s-1-\ell} g(T x+i)
$$

also has the property that $T^{d} \mid$ coeff $\left(x^{d}\right)$, for every monomial in $h(x) g(T x+i)$. This is true because $T^{d} \mid$ coeff $\left(x^{d}\right)$ in $g(T x+i)$, and in $h(n)$ we have $T^{k-s} \mid a_{k-s}$ by hypothesis and $b_{\ell} T^{\ell} \mid T^{\ell+1}$ for $b_{\ell} \mid T$, and thus $T^{k-s-(\ell+1)} \mid \frac{a_{k-s}}{b_{\ell} T^{\ell}}$.

We want to generalize Algorithm 1 to $\mathbf{Q P} \mathbf{P}_{\gg 0}$. First we need another expression for numerical polynomials.

Lemma 4.2. Let $f$ be a numerical polynomial, i.e., $f: \mathbf{Z} \rightarrow \mathbf{Z}$ with a polynomial $g(x) \in \mathbf{Q}[x]$ satisfying $f(n)=g(n)$ for all $n \in \mathbf{Z}$. Then there exists $T$, and $g_{i}(x) \in \mathbf{Z}[x]$ for $i=0, \ldots, T-1$ such that $f(n)=g_{i}(m)$ for all $n=T m+i$. 


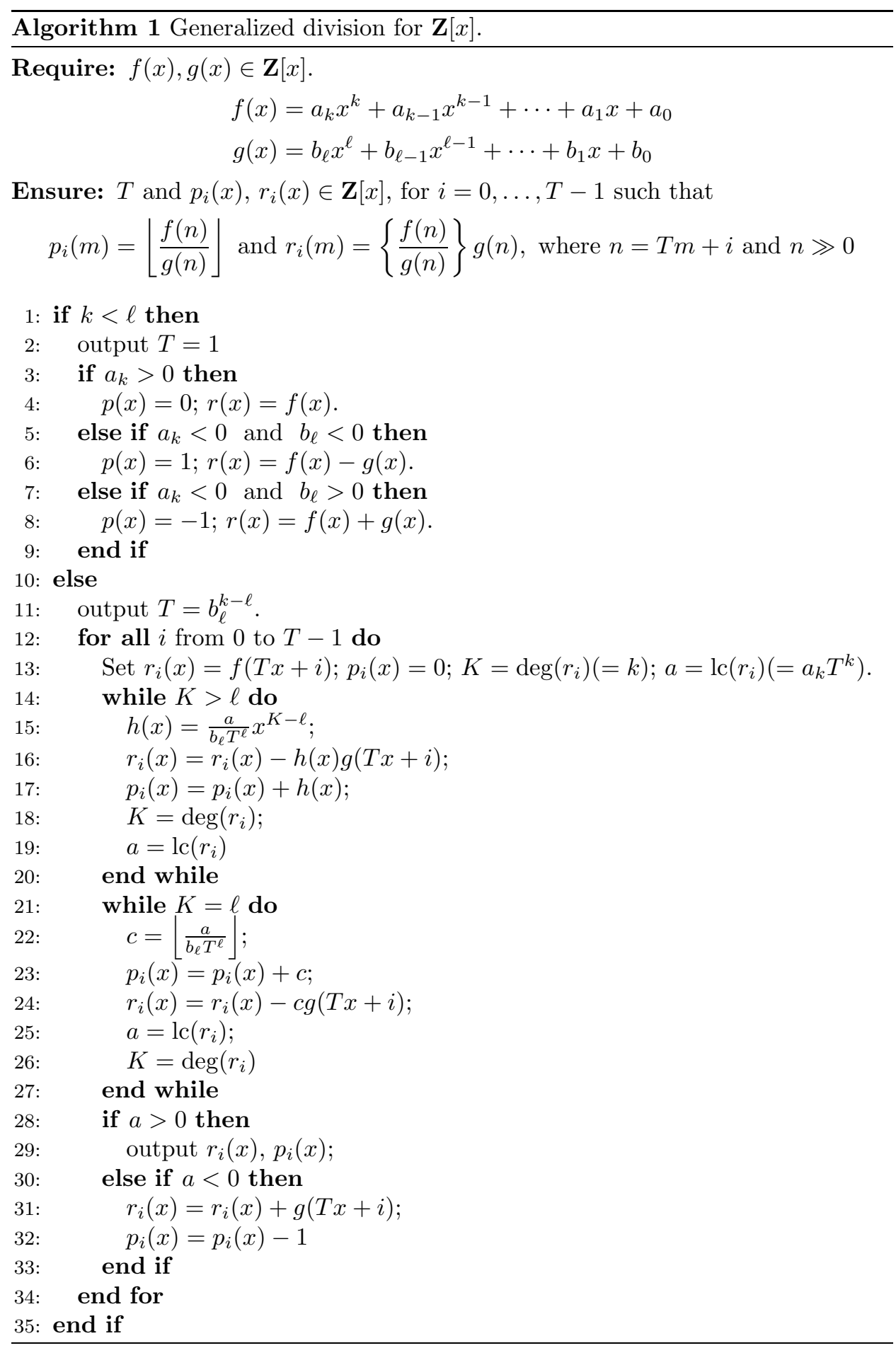


Proof. Suppose $\operatorname{Tg}(x) \in \mathbf{Z}[x]$ for some integer $T$. For $i=0, \ldots, T-1$, define $g_{i}(x)=g(T x+i)$. By the binomial expansion of $g(T x+i)-g(i)$, it is easy to see that this is a polynomial with integer coefficients.

Similarly, for an element $f \in \mathbf{Q P}_{\gg 0}$, there exists $T \in \mathbf{N}$ and $h_{i}(x) \in \mathbf{Z}[x]$ $(i=1, \ldots, T)$ satisfying $f(n)=h_{i}(m)$ for $n \gg 0$ and $n=T m+i$. We call $f=\left(T,\left\{h_{i}(x)\right\}_{i=1}^{T}\right)$ a representation of $f \in \mathbf{Q P}_{\gg 0}$. Another simple property is that for $f=\left(T_{1},\left\{f_{i}(x)\right\}_{i=1}^{T_{1}}\right)$ and $g=\left(T_{2},\left\{g_{i}(x)\right\}_{i=1}^{T_{2}}\right)$, we can extend to a common period $T=\operatorname{lcm}\left(T_{1}, T_{2}\right), f=\left(T,\left\{f_{i}^{\prime}(x)\right\}_{i=1}^{T}\right)$ and $g=\left(T,\left\{g_{i}^{\prime}(x)\right\}_{i=1}^{T}\right)$.

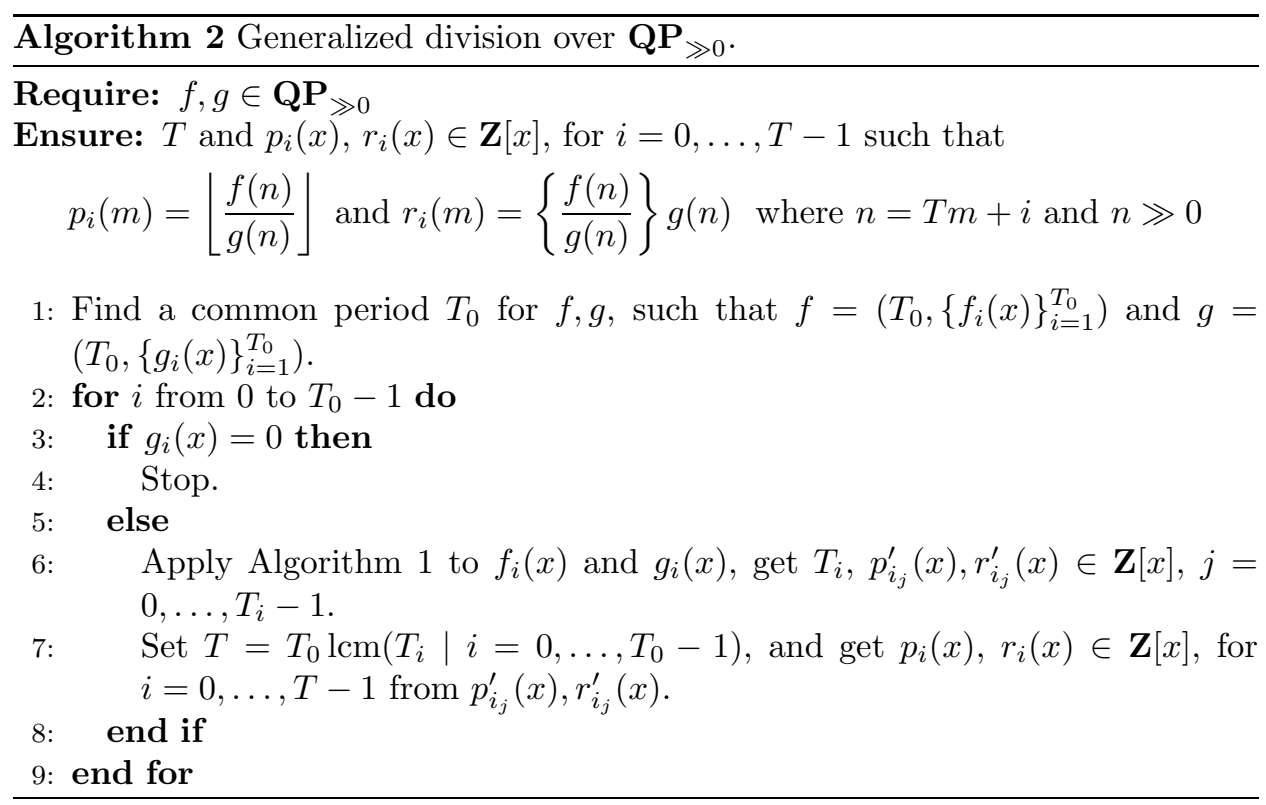

Theorem 4.3 (Generalized GCD). Let $f, g$ be numerical polynomials, or more generally $f, g \in \mathbf{Q P}_{\gg 0}$. Define $d(n)=\operatorname{gcd}(f(n), g(n))$. Then by the generalized division over $\mathbf{Q} \mathbf{P}_{\gg 0}$ (Algorithm 21), we have $d \in \mathbf{Q P}_{\gg 0}$. We call $d$ the generalized gcd of $f$ and $g$, and we denote it by $\operatorname{ggcd}(f, g)$.

Proof. Apply Algorithm 2 successively. Define $r_{-2}$ to be some constituent polynomial of $f$ and $r_{-1}$ to be some constituent polynomial of $g$ (a constituent polynomial of a quasi-polynomial is a polynomial on a congruence class modulo its period). In general, if $r_{i-1} \neq 0$, define $r_{i}$ to be some constituent polynomial of $\operatorname{rem}\left(r_{i-2}, r_{i-1}\right)$. We will prove that $r_{i}=0$ for some $i$ by induction on $\operatorname{deg}(r)$ and lc $(r)$. (Recall that lc $(r)$ means the leading coefficient of $r$.) Hence, since our choice of constituent polynomial was arbitrary, we will eventually have a remainder of 0 after finitely many steps. Hence the algorithm terminates after finitely many steps, or else there exists an infinite path in the decision tree. If $\operatorname{deg}\left(r_{i}\right)=0$ and $\operatorname{lc}\left(r_{i}\right)>0$, the algorithm works the same as the classical Euclidean algorithm for integers. If $\operatorname{deg}\left(r_{i}\right)>0$, then since $0 \leq r_{i+1}(n)<r_{i}(n)$ for $n \gg 0$, we have three cases for the next step:

1. $\operatorname{deg}\left(r_{i+1}\right)<\operatorname{deg}\left(r_{i}\right)$.

2. $\operatorname{deg}\left(r_{i+1}\right)=\operatorname{deg}\left(r_{i}\right)$ and $\operatorname{lc}\left(r_{i+1}\right)<\operatorname{lc}\left(r_{i}\right)$. After finitely many iterations, one will either have $\operatorname{lc}\left(r_{i+1}\right)=\operatorname{lc}\left(r_{i}\right)$ or $\operatorname{deg}\left(r_{i+1}\right)<\operatorname{deg}\left(r_{i}\right)$. 
3. $\operatorname{deg}\left(r_{i+1}\right)=\operatorname{deg}\left(r_{i}\right)$ and $\operatorname{lc}\left(r_{i+1}\right)=\operatorname{lc}\left(r_{i}\right)$. In the next step, $\operatorname{deg}\left(r_{i+2}\right)<$ $\operatorname{deg}\left(r_{i+1}\right)$.

So the algorithm terminates after finitely many steps.

Tracing through a natural generalization of the Euclidean algorithm, we can get $u, v \in \mathbf{Q P}_{\gg 0}$ such that $u f+v g=\operatorname{ggcd}(f, g)$. More generally, for $f_{1}, \ldots, f_{k} \in \mathbf{Q P}_{\gg 0}$ we have the following.

Corollary 4.4. Let $f_{1}, \ldots, f_{k} \in \mathbf{Q P}_{\gg 0}$ and $d=\operatorname{ggcd}\left(f_{1}, \ldots, f_{k}\right)$. Then $d \in \mathbf{Q P}_{\gg 0}$ and there exists $u_{1}, \ldots, u_{k} \in \mathbf{Q P}_{\gg 0}$ with $\operatorname{ggcd}\left(u_{1}, \ldots, u_{k}\right)=1$ such that

$$
f_{1} u_{1}+\cdots+f_{k} u_{k}=d .
$$

Remark 4.5. This implies, in particular, that every finitely generated ideal of $\mathbf{Q P} \mathbf{P}_{\gg 0}$ is principal. It is peculiar that in fact $\mathbf{Q} \mathbf{P}_{\gg 0}$ is not a Noetherian ring. For example, take $\chi_{d}$ to be the function such that $\chi_{d}(x)=1$ if $d \mid x$, and 0 otherwise. Then $I=\left(\chi_{2}, \chi_{4}, \chi_{8}, \ldots, \chi_{2^{n}}, \ldots\right)$ is not a finitely generated ideal.

Recall that for any $M \in \mathbf{Z}^{k \times s}$ there exist unimodular matrices $U, V$, i.e., determinant \pm 1 , such that $U M V=D=\left(\operatorname{diag}\left(d_{1}, \ldots, d_{r}, 0 \ldots, 0\right) \mid \mathbf{0}\right)$ with $d_{i} \mid d_{i+1}$, and $D$ is called the Smith normal form of $M$. By generalized division over $\mathbf{Q P} \mathbf{P}_{\gg 0}$ (Algorithm 2) and Corollary 4.4 we can generalize the classical Smith normal form theorem as follows.

Theorem 4.6. For any $M \in\left(\mathbf{Q P}_{\gg 0}\right)^{k \times s}$, define a matrix function $D: \mathbf{Z} \rightarrow \mathbf{Z}^{k \times s}$ such that $D(n)$ is the Smith normal form of $M(n)$. Then we have $D \in\left(\mathbf{Q P}_{\gg 0}\right)^{k \times s}$ and there exists $U \in\left(\mathbf{Q P}_{\gg 0}\right)^{k \times k}, V \in\left(\mathbf{Q P}_{\gg 0}\right)^{s \times s}$ such that $U(n)$ and $V(n)$ are unimodular for $n \gg 0$ and $U M V=D$. We call this matrix function $D$ the generalized Smith normal form of $M$.

Before the proof, let us see a simple example for a generalized gcd and a generalized Smith normal form.

Example 4.7. Let $f=2, g=x$. Denote $d=\operatorname{ggcd}(f, g)$, namely $d(n)=\operatorname{gcd}(2, n)$. Put $T=2$ and $d_{0}(x)=2, d_{1}(x)=1, u_{0}(x)=1, u_{1}(x)=-x, v_{0}(x)=0, v_{1}(x)=1$; we have

$$
d(n)=d_{i}(m)=u_{i}(m) f(n)+v_{i}(m) g(n) \text { for all } n=T m+i \text { and } i=0,1 .
$$

For $A=\left(\begin{array}{cc}2 & 3 x+2 \\ x & x^{3}+2 x\end{array}\right)$, let $U(x)=\left[U_{0}(x), U_{1}(x)\right]$, where

$$
U_{0}(x)=\left(\begin{array}{cc}
1 & 0 \\
-x & 1
\end{array}\right), \quad U_{1}(x)=\left(\begin{array}{cc}
-x & 1 \\
-2 x-1 & 2
\end{array}\right)
$$

$V(x)=\left[V_{0}(x), V_{1}(x)\right]$, where

$$
V_{0}(x)=\left(\begin{array}{cc}
1 & -(3 x+1) \\
0 & 1
\end{array}\right), \quad V_{1}(x)=\left(\begin{array}{cc}
1 & -\left(8 x^{3}+6 x^{2}+5 x+3\right) \\
0 & 1
\end{array}\right),
$$

and $D(x)=\left[D_{0}(x), D_{1}(x)\right]$, where

$$
D_{0}(x)=\left(\begin{array}{cc}
2 & 0 \\
0 & 8 x^{3}-6 x^{2}+2 x
\end{array}\right), \quad D_{1}(x)=\left(\begin{array}{cc}
1 & 0 \\
0 & 16 x^{3}+12 x^{2}+4 x+1
\end{array}\right) .
$$

We have that $U_{i}(m) A(n) V(m)=D_{i}(m)$ is the Smith normal form for $A(n)$ for $n \gg 0$ where $n=2 m+i$ and $i=0,1$. 
The following proof is based on the proof for a classical Smith normal form in New.

Lemma 4.8. Let $\alpha_{1}, \alpha_{2}, \ldots, \alpha_{k} \in \mathbf{Q P}_{\gg 0}$ with $\operatorname{ggcd}\left(\alpha_{1}, \alpha_{2}, \ldots, \alpha_{k}\right)=d_{k}$. Then there is a matrix $W_{k} \in\left(\mathbf{Q P}_{\gg 0}\right)^{k \times k}$ with first row $\left[\alpha_{1}, \alpha_{2}, \ldots, \alpha_{k}\right]$ and determinant $d_{k}$.

Proof. By induction on $k$. For $k=1$, trivial. Now suppose the lemma is true for $k=n-1$, where $n \geq 2$, and let $W_{k-1} \in\left(\mathbf{Q P}_{\gg 0}\right)^{(k-1) \times(k-1)}$ be a matrix with first row $\left[\alpha_{1}, \alpha_{2}, \ldots, \alpha_{k-1}\right]$ and determinant $d_{k-1}=\operatorname{ggcd}\left(\alpha_{1}, \alpha_{2}, \ldots, \alpha_{k-1}\right)$. Since

$$
\begin{aligned}
d_{k} & =\operatorname{ggcd}\left(\alpha_{1}, \alpha_{2}, \ldots, \alpha_{k}\right) \\
& \left.=\operatorname{ggcd}\left(\alpha_{1}, \alpha_{2}, \ldots, \alpha_{k-1}\right), \alpha_{k}\right) \\
& =\operatorname{ggcd}\left(d_{k-1}, \alpha_{k}\right),
\end{aligned}
$$

by Corollary 4.4, there exist $\rho, \sigma \in \mathbf{Q P}_{\gg 0}$ such that $\rho d_{k-1}-\sigma \alpha_{k}=d_{k}$. Put

$$
W_{k}=\left(\begin{array}{ccccc} 
& & & & \alpha_{k} \\
& W_{k-1} & & & 0 \\
& & & & \vdots \\
\frac{\alpha_{1} \sigma}{d_{k-1}} & \frac{\alpha_{2} \sigma}{d_{k-1}} & \ldots & \frac{\alpha_{k-1} \sigma}{d_{k-1}} & \rho
\end{array}\right) .
$$

Then we have $W_{k} \in\left(\mathbf{Q P}_{\gg 0}\right)^{k \times k}$ with first row $\left[\alpha_{1}, \alpha_{2}, \ldots, \alpha_{k}\right]$ and $\operatorname{det}\left(W_{k}\right)=$ $\rho d_{k-1}-\sigma \alpha_{k}=d_{k}$.

Proof of Theorem 4.6. Write $A=\left(a_{i j}\right) \neq 0 \in\left(\mathbf{Q P}_{\gg 0}\right)^{k \times s}$. If $a_{11} \neq 0$, then switch rows and columns so that this is true. Without loss of generality, we may assume that $a_{11}$ divides all elements in the column. If not, let $d=\operatorname{ggcd}\left(a_{11}, \ldots, a_{k 1}\right)$. By Corollary 4.4, there exist $u_{1}, \ldots, u_{k} \in \mathbf{Q} \mathbf{P}_{\gg 0}$ with $\operatorname{ggcd}\left(u_{1}, \ldots, u_{k}\right)=1$ such that $u_{1} a_{11}+\cdots+u_{k} a_{k 1}=d$. By Lemma 4.8, there exists a matrix $W \in\left(\mathbf{Q P}_{\gg 0}\right)^{k \times k}$ with first row $\left[u_{1}, \ldots, u_{k}\right]$ and determinant 1 . So we have $A^{\prime}=\left(a_{i j}^{\prime}\right)=W A$ such that $a_{11}^{\prime}$ divides all elements in the column. Suppose $a_{11}$ divides all elements $a_{i j}$. First, by the same process as above, we can assume that $a_{11}$ divides all elements in the first row and column. By elementary row and column operations, all the elements in the first row and column, other than the $(1,1)$ position, can be made zero. If there is some element $a_{i j}$, where $i, j \neq 1$ is not divisible by $a_{11}$, then add column $j$ to column 1 and repeat the previous step to replace $a_{11}$ with one of its divisors which divides $a_{i j}$. Thus we must finally reach the stage where the element in the $(1,1)$ position divides every element of the matrix and where all the other elements of the first row and column are zero. Repeat the entire process with the submatrix obtained by deleting the first row and column.

\section{Application of generalized division in the COMputation OF SOME GENERALIZED EHRHART POLYNOMIALS}

We can see that the algorithm we give in the proof of Theorem 1.1 is not very efficient. In this section, by the theory of generalized division developed in the previous section, we are able to compute some special cases more efficiently. 
5.1. Generalized Popoviciu formula. First recall the classical Popoviciu formula. Let $a$ and $b$ be relatively prime positive integers. Then the number of nonnegative integer solutions $(x, y)$ to the equation $a x+b y=n$ is given by the formula (1.1). Now consider the equation

$$
a(n) x+b(n) y=m(n),
$$

where $a(n), b(n), m(n)$ are integeral polynomials of $n$. By the theory of generalized division, the above formula for the number of nonnegative integer solutions $p_{\left\{a_{1}(n), a_{2}(n)\right\}}(m(n))$ can be easily generalized to the following.

Proposition 5.1. Suppose $\operatorname{ggcd}(a(n), b(n))=1$. Then

$$
p_{\left\{a_{1}(n), a_{2}(n)\right\}}(m(n))=\frac{m(n)}{a_{1}(n) a_{2}(n)}-\left\{\frac{a_{1}(n)^{-1} m(n)}{a_{2}(n)}\right\}-\left\{\frac{a_{2}(n)^{-1} m(n)}{a_{1}(n)}\right\}+1,
$$

where $a_{1}(n) a_{1}(n)^{-1}+a_{2}(n) a_{2}(n)^{-1}=1$. In particular, $p_{\left\{a_{1}(n), a_{2}(n)\right\}}(m(n)) \in$ $\mathbf{Q P}_{\gg 0}$.

If $\operatorname{ggcd}(a(n), b(n))=d(n) \neq 1$, we can simply divide it from both sides and reduce to the case of $\operatorname{ggcd}(a(n), b(n))=1$.

Then as mentioned in the introduction, we can consider the number of solutions $(x, y, z) \in \mathbf{Z}_{\geq 0}^{3}$ to the matrix equation (1.2), where the $x_{i}$ and $y_{i}$ are fixed positive integers and $x_{i+1} y_{i}<x_{i} y_{i+1}$ for $i=1,2$. Write $Y_{i j}=x_{i} y_{j}-x_{j} y_{i}$. We assume that $\operatorname{gcd}\left(Y_{12}, Y_{13}, Y_{23}\right)=1$ so that there exist integers (not unique) $f_{i j}, g_{i j}$ such that

$$
\begin{aligned}
& \operatorname{gcd}\left(f_{12} Y_{13}+g_{12} Y_{23}, Y_{12}\right)=1, \\
& \operatorname{gcd}\left(f_{13} Y_{12}+g_{13} Y_{23}, Y_{13}\right)=1, \\
& \operatorname{gcd}\left(f_{23} Y_{13}+g_{23} Y_{12}, Y_{23}\right)=1 .
\end{aligned}
$$

Now define two regions $\Omega_{i}=\left\{(x, y) \mid \frac{y_{i}}{x_{i}}<\frac{y}{x}<\frac{y_{i+1}}{x_{i+1}}\right\}$ for $i=1,2$. Then if $m=\left(m_{1}, m_{2}\right) \in \mathbf{Z}^{2}$ is in the positive span of the columns of the matrix in (1.2), there exist the following Popoviciu-like formulas [Xu, Theorem 4.3] for $t(m \mid A)$, the number of solutions of (1.2).

When $m=\left(m_{1}, m_{2}\right)^{T} \in \bar{\Omega}_{1} \cap \mathbf{Z}^{2}$,

$$
\begin{aligned}
t(m \mid A)= & \frac{m_{2} x_{1}-m_{1} y_{1}}{Y_{12} Y_{13}}+1 \\
& -\left\{\frac{\left(f_{12} Y_{13}+g_{12} Y_{23}\right)^{-1}\left(m_{2}\left(f_{12} x_{1}+g_{12} x_{2}\right)-m_{1}\left(f_{12} y_{1}+g_{12} y_{2}\right)\right)}{Y_{12}}\right\} \\
& -\left\{\frac{\left(f_{13} Y_{12}+g_{13} Y_{23}\right)^{-1}\left(m_{2}\left(f_{13} x_{1}+g_{13} x_{3}\right)-m_{1}\left(f_{13} y_{1}+g_{13} y_{3}\right)\right)}{Y_{13}}\right\} .
\end{aligned}
$$

When $m=\left(m_{1}, m_{2}\right)^{T} \in \bar{\Omega}_{2} \cap \mathbf{Z}^{2}$,

$$
\begin{aligned}
t(m \mid A)= & \frac{m_{1} y_{3}-m_{2} y_{3}}{Y_{23} Y_{13}}+1 \\
& -\left\{\frac{\left(f_{23} Y_{13}+g_{23} Y_{12}\right)^{-1}\left(m_{1}\left(f_{23} x_{3}+g_{23} x_{2}\right)-m_{2}\left(f_{23} y_{3}+g_{23} y_{2}\right)\right)}{Y_{23}}\right\} \\
& -\left\{\frac{\left(f_{13} Y_{12}+g_{13} Y_{23}\right)^{-1}\left(m_{1}\left(f_{13} x_{1}+g_{13} x_{3}\right)-m_{2}\left(f_{13} y_{1}+g_{13} y_{3}\right)\right)}{Y_{13}}\right\},
\end{aligned}
$$


where $f_{12}, g_{12}, f_{13}, g_{13}, f_{23}$ and $g_{23} \in \mathbf{Z}$ satisfy

$$
\begin{aligned}
\operatorname{gcd}\left(f_{12} Y_{13}+g_{12} Y_{23}, Y_{12}\right) & =\operatorname{gcd}\left(f_{13} Y_{12}+g_{13} Y_{23}, Y_{13}\right) \\
& =\operatorname{gcd}\left(f_{23} Y_{13}+g_{23} Y_{12}, Y_{23}\right)=1
\end{aligned}
$$

and

$$
\begin{array}{ll}
\left(f_{12} Y_{13}+g_{12} Y_{23}\right)^{-1}\left(f_{12} Y_{13}+g_{12} Y_{23}\right) \equiv 1 & \left(\bmod Y_{12}\right), \\
\left(f_{13} Y_{12}+g_{13} Y_{23}\right)^{-1}\left(f_{13} Y_{12}+g_{13} Y_{23}\right) \equiv 1 & \left(\bmod Y_{13}\right), \\
\left(f_{23} Y_{13}+g_{23} Y_{12}\right)^{-1}\left(f_{23} Y_{13}+g_{23} Y_{12}\right) \equiv 1 & \left(\bmod Y_{23}\right) .
\end{array}
$$

Notice that in the above formula everything can be directly generalized to the ring $\mathbf{Z}[x]$ using generalized division and generalized GCD. In other words, we can replace the $x_{i}, y_{i}$, and $m_{i}$ by polynomials in $n$ in such a way that for all values of $n$, the condition $\operatorname{gcd}\left(Y_{12}, Y_{13}, Y_{23}\right)=1$ always holds. For example, consider the system

$$
\left(\begin{array}{ccc}
2 n+1 & 3 n+1 & n^{2} \\
2 & 3 & n+1
\end{array}\right)\left(\begin{array}{l}
x \\
y \\
z
\end{array}\right)=\left(\begin{array}{c}
3 n^{3}+1 \\
3 n^{2}+n-1
\end{array}\right) .
$$

Then for $n \gg 0$, we have that

$$
\frac{3}{3 n+1}<\frac{3 n^{2}+n-1}{3 n^{3}+1}<\frac{n+1}{n^{2}},
$$

so the number of solutions $(x, y, z)$ is counted by the formula of $t(m \mid A)$ for $m \in \bar{\Omega}_{2}$ and it is easy to see that $t(m \mid A) \in \mathbf{Q P}_{\gg 0}$.

5.2. Dimension two. For polytopes in dimension two, there is an efficient algorithm for computing the number of lattice points using continued fractions. See Bar, Chapter 15] for the algorithm in the case of nonparametrized two dimensional polytopes.

By the generalized division theory, we can generalize this algorithm to the case of a polytope whose vertices are given by polynomials. Notice that the only difference is the generalization of continued fractions: for any $f, g \in \mathbf{Z}[x]$, we expand $f / g$ as a continued fraction. Then by Theorem 4.3, for $n \gg 0$, the terms in the expansion are quasi-polynomials and the number of terms does not depend on $n$.

We use the following notation for continued fractions. First, set $[a]=a$, and in general, we set

$$
\left[a_{0} ; a_{1}, \ldots, a_{k}\right]=a_{0}+\frac{1}{\left[a_{1} ; a_{2}, \ldots, a_{k}\right]} .
$$

Example 5.2. For $n>4$, we have

$$
\frac{n^{2}}{2 n+1}= \begin{cases}{[m-1 ; 1,3, m],} & n=2 m, \\ {[m-1 ; 3,1, m-1],} & n=2 m-1 .\end{cases}
$$


Also, for the cone generated by $(0,1)$ and $\left(2 n+1, n^{2}\right)$, we have the following efficient algorithm of decomposition into prime cones:

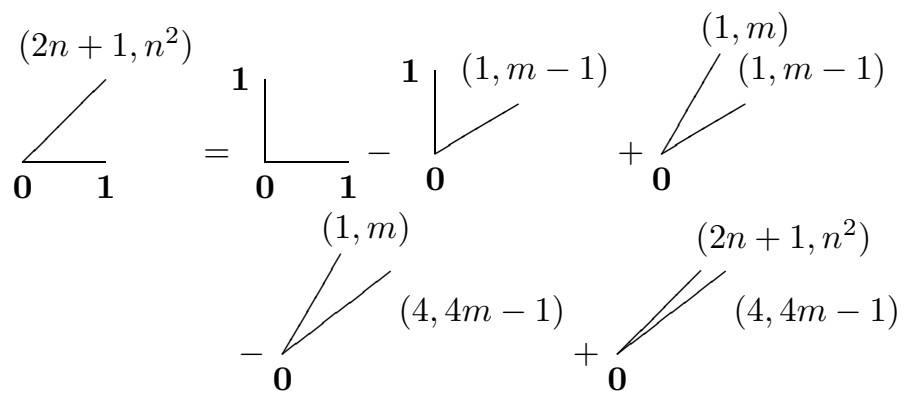

\section{ACKNOWLEDGEMENTS}

We thank Richard Stanley for useful discussions and for reading previous drafts of this article. We also thank an anonymous referee for helping to improve the quality and readability of the paper.

\section{REFERENCES}

[Bar] Alexander Barvinok, Integer Points in Polyhedra, Zürich Lectures in Advanced Mathematics, European Mathematical Society, Zürich, 2008. MR2455889

[BR] Matthias Beck and Sinai Robins, Computing the Continuous Discretely: Integer-point enumeration in polyhedra, Undergraduate Texts in Mathematics, Springer, New York, 2007, available for download from http://math.sfsu.edu/beck/ccd.html. MR2271992 (2007h:11119)

[Ehr] E. Ehrhart, Polynômes arithmétiques et Méthode des Polyèdres en Combinatoire, International Series of Numerical Mathematics, vol. 35, Birkhäuser Verlag, Basel/Stuttgart, 1977. MR0432556 (55:5544)

[New] Morris Newmann, Integral Matrices, Academic Press, New York, 1972. MR0340283 (49:5038)

[Sta] Richard P. Stanley, Enumerative Combinatorics, Vol. I, Cambridge Studies in Advanced Mathematics 49, Cambridge University Press, 1997. MR.1442260 (98a:05001)

$[\mathrm{Xu}]$ Zhiqiang $\mathrm{Xu}$, An explicit formulation for two dimensional vector partition functions, Integer Points in Polyhedra-Geometry, Number Theory, Representation Theory, Algebra, Optimization, Statistics, Contemporary Mathematics 452 (2008), 163-178. MR 2405771 (2009h:11045)

Department of Mathematics, Harbin Institute of Technology, Harbin, People's RePUBLIC OF CHINA 150001

E-mail address: schen@hit.edu.cn

Department of Mathematics, Massachusetts Institute of Technology, Cambridge, Massachusetts 02139

E-mail address: nan@math.mit.edu

Department of Mathematics, Massachusetts Institute of Technology, Cambridge, MassaChusetts 02139

E-mail address: ssam@math.mit.edu 